\title{
Aplicação do TOPSIS na análise do processo de infusão a vácuo para fabricação de compósitos com fios de juta
}

\author{
Alessandro de Castro Corrêa ${ }^{1 *}$ (1) \\ Jean da Silva Rodrigues ${ }^{1}$ \\ Tainã Fernandes Rodrigues ${ }^{1}$ \\ Carlos André Corrêa de Mattos ${ }^{2}$ \\ Danielle Cristina Gonzaga Corrêa ${ }^{3}$ \\ Cláudia Canto de Souza Leão ${ }^{1}$
}

\section{Resumo}

O objetivo deste estudo foi analisar o desempenho do processo de infusão a vácuo (VIP) na fabricação de compósitos de fios de juta e resina poliéster em comparação ao processo de laminação manual (hand lay-up), considerando múltiplos atributos com apoio do Technique for Order of Preference by Similarity to Ideal Solution (TOPSIS). O experimento envolveu a produção de compósitos de matriz de poliéster insaturado reforçados por fios de juta na forma de tecido plano. As medidas de desempenho foram a porosidade, resistência à tração, custos, redução de emissão da voláteis e a expectativa de contribuição social. Os pesos dos atributos foram determinados objetivamente a partir da informação disponível em cada critério na matriz de decisão com base na entropia da informação. O processo de laminação manual foi utilizado como referência de avaliação por ser considerado o processo convencional. Os resultados revelaram que os atributos com maior peso foram a redução de emissão de voláteis e a porosidade; que o processo VIP apresentou um desempenho superior ao processo hand lay-up; e que a TOPSIS foi apropriada a análise do problema.

Palavras-chave: Infusão a vácuo; TOPSIS; Entropia; Compósitos; Fios de juta.

\section{Application of TOPSIS in evaluating the vacuum infusion process toward manufacturing of jute yarn composites}

\begin{abstract}
This study aims to compare the vacuum infusion process (VIP) to the conventional hand lay-up process (HLU) applied to the manufacturing of jute yarn and polyester resin composites, considering multiple attributes with the assistance of the Technique for Order of Preference by Similarity to Ideal Solution (TOPSIS). The experiment consisted of producing unsaturated polyester composites reinforced by jute yarn, arranged in the form of fabric in plan style. The attributes considered were porosity, tensile strength, costs, reduction of volatile emissions, and social perspective. Shannon's entropy was applied to determine attributes weights objectively. HLU was selected as the benchmark because it is considered the conventional process. The results obtained show that reduction of volatile emissions and tensile strength were the weightiest attributes, the VIP was the best choice, and TOPSIS was appropriate for the problem analysis.
\end{abstract}

Keywords: Vacuum infusion process; TOPSIS; Entropy; Composite; Jute yarn.

\section{Introdução}

As decisões relativas à seleção de processos de produção de materiais envolvem a consideração de diversos atributos que podem ser de difícil mensuração, qualitativos e antagônicos.
Os engenheiros, como tomadores de decisões, têm se deparado com problemas cada vez mais complexos, por um lado, em decorrência das crescentes exigências ambientais e sociais,

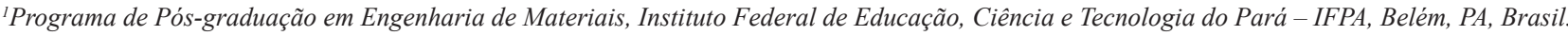

${ }^{2}$ Faculdade de Administração, Universidade Federal do Pará - UFPA, Belém, PA, Brasil.

${ }^{3}$ Instituto de Desenvolvimento Florestal e da Biodiversidade do Estado do Pará-IDEFLOR-Bio, Belém, PA, Brasil.

*Autor correspondente: alessandro.correa4@gmail.com

2176-1523 (C) 2021. Corrêa et al. Publicado pela ABM. Este é um artigo publicado em acesso aberto (Open Access) sob a licença Creative Commons Attribution, que permite uso, distribuição e reprodução em qualquer meio, sem restrições desde que o trabalho original seja Commons Attribution,
corretamente citado. 
por outro, por causa das novas opções que o desenvolvimento tecnológico tem disponibilizado. Assim, mesmo após o atendimento de requisitos técnicos, não é raro que se depare com um conjunto de alternativas com variados atributos, tornando a sua seleção uma tarefa nada trivial.

No contexto de materiais, um processo pode ser definido como "um método de conformação, união ou acabamento de um material" [1]. Segundo Levy e Pardini [2], o processo de fabricação corresponde de 50 a $60 \%$ do custo total de um compósito, sendo, portanto, fundamental a seleção de um processo adequado e suficiente para atender aos parâmetros de produção requeridos.

O processo de infusão a vácuo (Vacuum Infusion Process - VIP) envolve a infusão de uma resina de baixa viscosidade, numa preforma fibrosa e seca, que é colocada num molde rígido e coberta por uma membrana flexível. O processo se baseia num gradiente de pressão para conduzir a resina para dentro do molde e impregnar a preforma. Ragondet [3] esclarece que a aceitação do VIP se deve a sua baixa emissão de compostos orgânicos voláteis, baixo custo do conjunto ferramental, bem como a sua maior reprodutibilidade, em decorrência do menor envolvimento do operador, quando comparado com as técnicas de molde aberto, como hand lay-up (HLU).

A avaliação de processos candidatos, bem como de materiais para uma determinada aplicação, envolve a interpretação dos requisitos do projeto, a triagem e a classificação das alternativas restantes, envolvendo diversos atributos que podem ser conflitantes entre si, tornando o problema de seleção uma atividade complexa. Nesses cenários, os métodos de Apoio Multicritério à Decisão (AMD) têm contribuído para sistematizar o processo de análise de dados e melhorado o processo decisório, promovendo um crescente interesse pela sua aplicação. Dentre esses métodos, está o Technique for Order of Preference by Similarity to Ideal Solution (TOPSIS), proposto por Hwang e Yoon [4] que se destaca pela simplicidade de cálculo, por permitir a atribuição subjetiva dos pesos dos atributos e pela capacidade de considerar riscos envolvidos [5].

Desta forma, o objetivo do presente estudo foi analisar o desempenho do processo de infusão a vácuo (VIP) na produção de compósitos fios de juta e resina poliéster em comparação ao processo hand lay-up (HLU), considerando múltiplos atributos com apoio do Método TOPSIS.

\section{TOPSIS}

Os métodos AMD constituem um conjunto de técnicas destinadas à assistência no processo de tomada de decisões complexas, envolvendo diversos aspectos, os quais, mesmo que sejam conflituosos, devem ser avaliados conjuntamente. Podem ser classificados em métodos Multiatributos de Tomada de Decisão (Multi-Atttribute Decision Making - MADM) e Métodos Multiobjetivos de Tomada de Decisão (Multiple Objective Decision Making - MODM) [4]. Enquanto os Métodos MADM consideram um número limitado e discreto de alternativas avaliadas com base em vários atributos, com níveis de importância variadas; nos métodos MODM, as variáveis de decisão são definidas num domínio inteiro ou discreto com um número grande ou infinito de alternativas de escolha, envolvendo restrições [5].

O Technique for Order of Preference by Similarity to Ideal Solution (TOPSIS) é um dos MADM mais populares e classifica as alternativas por sua proximidade da solução ideal (ou positiva ou desejada) e distanciamento da solução antiideal (ou negativa ou não desejada), escolhendo a alternativa com maiores benefícios, o que significa que procura evitar o risco de seleção de alternativas que reduzam os resultados tanto quanto possível.

O TOPSIS vem sendo aplicado em variados problemas de seleção de materiais na engenharia, como se pode observar nos estudos apresentados na Tabela 1. Por vezes, é utilizado como o único método de avaliação [6-8,15-17]. Em outros estudos, para fins de comparação, como um dentre outros métodos de seleção [9-14]. O método também vem sendo aplicado especificamente na seleção de materiais compósitos reforçados com fibras naturais $[15,16]$ e na determinação da composição ótima de compósitos reforçados por fibras naturais [17].

No tocante à designação dos pesos dos atributos, podem-se observar três situações: a primeira, quando o analista é capaz de determinar diretamente os seus valores relativos $[7,11,12,15]$; a segunda, quando estudos recorrem à integração com outros métodos com o propósito de apoiar a designação dos pesos, optando por métodos que se baseiam nas preferências expressadas pelos tomadores por meio de comparações pareadas [8,9,13-16], destacando-se nesse caso o Analytic Hierarchy Process (AHP), método proposto por Saaty [18]; por fim, a terceira situação, quando, na impossibilidade de obter tais preferências, a Entropia da Informação [19] é uma alternativa capaz de extrair objetivamente os pesos conforme a quantidade de informação contida em cada atributo sem necessidade de intervenção do tomador de decisão.

As etapas do método são as seguir descritas.

$1^{\text {a }}$ etapa: Inicialmente, os atributos destinados à avaliação das alternativas devem ser definidos, bem como as suas importâncias relativas de forma a refletirem as preferências do decisor para a aplicação em questão.

$\mathbf{2}^{\mathbf{a}}$ etapa: Em seguida, coletam-se as medidas de desempenho em cada atributo das $m$ alternativas, organizando-as numa matriz de decisão como a descrita a seguir (Equação 1):

$$
A=\left[x_{i j}\right]_{m \times n}
$$

em que $x_{i j}$ corresponde ao desempenho, ou utilidade, da alternativa $i$ associado ao atributo $j, i=1, \ldots, m$ e $j=1, \ldots, n$.

$3^{\text {a }}$ etapa: Normalizam-se as medidas de desempenho, para que sejam eliminadas as diferenças de escalas entre as medidas, por meio da Equação 2: 
Tabela 1. Aplicações do TOPSIS em problemas de engenharia de materiais

\begin{tabular}{|c|c|c|c|}
\hline Autoria & Métodos & Aplicação & Pesos \\
\hline Milani et al. [6] & TOPSIS & $\begin{array}{l}\text { Seleção de material de engrenagem de } \\
\text { transmissão }\end{array}$ & Entropia \\
\hline Shanian e Savadogo [7] & TOPSIS & $\begin{array}{c}\text { Seleção de materiais para placas metálicas } \\
\text { bipolares destinadas a células de combustíveis } \\
\text { poliméricas. }\end{array}$ & \\
\hline Govindan et al. [8] & TOPSIS & $\begin{array}{l}\text { Seleção de materiais sustentáveis para } \\
\text { construção. }\end{array}$ & DEMATEL; ANP \\
\hline Shanian e Savadogo [9] & TOPSIS; VIKOR; ELECTRE & $\begin{array}{l}\text { Seleção de componentes térmicos para a } \\
\text { indústria aeroespacial e nuclear. }\end{array}$ & FAHP \\
\hline Chauhan e Vaish [10] & TOPSIS; VIKOR & $\begin{array}{c}\text { Seleção de materiais magnéticos para aplicação } \\
\text { em capacitores. }\end{array}$ & Entropia \\
\hline Chauhan e Vaish [11] & $\begin{array}{l}\text { TOPSIS; DI-TOPSIS; I-TOPSIS; } \\
\text { I-VIKOR; Cross entropy; }\end{array}$ & $\begin{array}{c}\text { Seleção de materiais magnéticos para aplicação } \\
\text { em capacitores. }\end{array}$ & \\
\hline Karande e Chakraborty [12] & $\begin{array}{l}\text { MOORA; MULTIMOORA; Reference } \\
\text { point approach }\end{array}$ & $\begin{array}{l}\text { Seleção de materiais volante de motor, tanque } \\
\text { para nitrogênio líquido e mastro de barco à vela. }\end{array}$ & \\
\hline Çalışkan et al. [13] & TOPSIS; EXPROM2; VIKOR & Seleção de materiais para fixador de ferramenta & Entropia; AHP \\
\hline Anojkumar et al. [14] & $\begin{array}{l}\text { TOPSIS; VIKOR; ELECTRE I; } \\
\text { PROMETHEE; FUZZY }\end{array}$ & $\begin{array}{l}\text { Seleção de material para tubos metálicos } \\
\text { destinados à indústria de açúcar. }\end{array}$ & FAHP \\
\hline Mansor et al. [15] & TOPSIS & $\begin{array}{c}\text { Seleção de materiais de matriz termofixa para } \\
\text { bio-compósitos híbridos para para-choques } \\
\text { automotivos. }\end{array}$ & \\
\hline Mansor et al. [16] & TOPSIS & $\begin{array}{l}\text { Seleção de materiais compósitos híbridos de } \\
\text { fibras naturais destinados a componente de freio } \\
\text { de mão automotivo. }\end{array}$ & AHP \\
\hline Al-Oqla et al. [17] & TOPSIS & $\begin{array}{l}\text { Composição ótima de compósitos reforçados } \\
\text { por fibras naturais com base em propriedades } \\
\text { de tração. }\end{array}$ & AHP \\
\hline
\end{tabular}

Nota: ANP = Analytical Network Process; DEMATEL = Decision Making Trail and Evaluation Laboratory; DI = Direct Interval; ELECTRE = Elimination Et Choix Traduisant La Realite; EXPROM2 = Extended Preference Ranking Organization Method for Enrichment Evaluation; FAHP = Fuzzy Analytical Hierarchy Process; I = Interval data; MOORA = Multi-Objective Optimization on the basis of Ratio Analysis; MULTIMOORA = Multi-Objective Optimization by Ratio Analysis plus the Full Multiplicative Form; PROMETHEE = Preference Ranking Organization Method for Enrichment Evaluation; e VIKOR = Vise Kriterijumsa Optimizacija I Kompromisno Resenje.

$$
r_{i j}=\frac{x_{i j}}{\sqrt{\sum_{i=I}^{m} x_{i j}^{2}}}
$$

na qual $r_{i j}$ são as medidas de desempenho $x_{i j}$ normalizadas.

$4^{\text {a }}$ etapa: Ponderaram-se os valores normalizados pelos pesos dos respectivos atributos.

$$
v_{i j}=w_{j} r_{i j}
$$

onde $w_{j}$ é o peso do atributo $j$.

$5^{\text {a }}$ etapa: Identificam-se as soluções ideais $\left(A^{+}\right)$e antiideais ( $\left.A^{-}\right)$, conforme Equações 4 e 5 :

$$
\begin{aligned}
& A^{+}=\left\{v_{1}^{+}, v_{2}^{+}, \ldots, v_{j}^{+}, \ldots v_{n}^{+}\right\}=\left\{\left(\max _{i} v_{i j} \mid j \in J_{I}\right),\left(\min _{i} v_{i j} \mid j \in J_{2}\right) \mid i=1, \ldots, m\right\} \\
& A^{-}=\left\{v_{1}^{-}, v_{2}^{-}, \ldots, v_{j}^{-}, \ldots v_{n}^{-}\right\}=\left\{\left(\min _{i} v_{i j} \mid j \in J_{1}\right),\left(\max _{i} v_{i j} \mid j \in J_{2}\right) \mid i=1, \ldots, m\right\}
\end{aligned}
$$

onde $J_{1}$ é o conjunto de atributos que correspondem aos benefícios, geralmente o maior valor observado entre as alternativas, e $J_{2}$ é o conjunto de atributos de custos ou resultados que pretende minimizar, em geral, o menor valor.

$6^{\text {a }}$ etapa: São calculadas as distâncias de cada alternativa $i$ das soluções ideais $\left(s_{i}^{+}\right)$e anti-ideais $\left(s_{i}^{-}\right)$, utilizando-se a distância Euclidiana, conforme Equações 6 e 7:

$$
s_{i}^{+}=\sqrt{\sum_{i=1}^{n}\left(v_{i j}-v_{i}^{+}\right)^{2}}
$$

$$
s_{i}^{-}=\sqrt{\sum_{i=1}^{n}\left(v_{i j}-v_{i}^{-}\right)^{2}}
$$

$7^{\text {a }}$ etapa: Finalmente, calcula-se a medida de proximidade $\left(C_{i}\right)$ de cada alternativa, conforme a Equação 8, que representa a sua pontuação. As alternativas, então, podem ser ranqueadas seguindo o critério de quanto maior, melhor a alternativa.

$$
C_{i}=\frac{s_{i}^{-}}{s_{i}^{+}+s_{i}^{-}}
$$




\section{Materiais e métodos}

Para analisar o desempenho da fabricação de compósitos de fibra de juta pelo processo de infusão a vácuo (VIP), realizaram-se os procedimentos listados no fluxograma apresentado na Figura 1. Inicialmente é importante que se estabeleça uma referência de desempenho, uma vez que para que um processo produtivo seja selecionado não basta que seja somente viável, mas precisa ser superior à alternativa correntemente utilizada. Escolheu-se o processo de hand lay-up (HLU) para ser a referência de comparação (benchmark) na análise, tendo em vista que atualmente é considerado como uma maneira convencional de fabricação de compósitos reforçados por fibras de juta para peças similares.

Em seguida, foram determinados os atributos que devem refletir os desempenhos de interesse para a avaliação.

Tabela 2. Atributos dos processos e suas características

\begin{tabular}{lccc}
\hline \multicolumn{1}{c}{ Atributos } & Tipo & Direção & Unidade \\
\hline 1 Porosidade & Quantitativo & Minimizar & Proporção \\
$\begin{array}{l}\text { 2 Resistência à } \\
\text { tração }\end{array}$ & Quantitativo & Maximizar & $\mathrm{MPa}$ \\
3 Custos & Quantitativo & Minimizar & $\mathrm{R} \$$ \\
4 Redução da & Qualitativo & Maximizar & Saaty \\
emissão de voláteis & & & \\
5 Expectativa de & Qualitativo & Maximizar & Saaty \\
contribuição social & & & \\
\hline
\end{tabular}

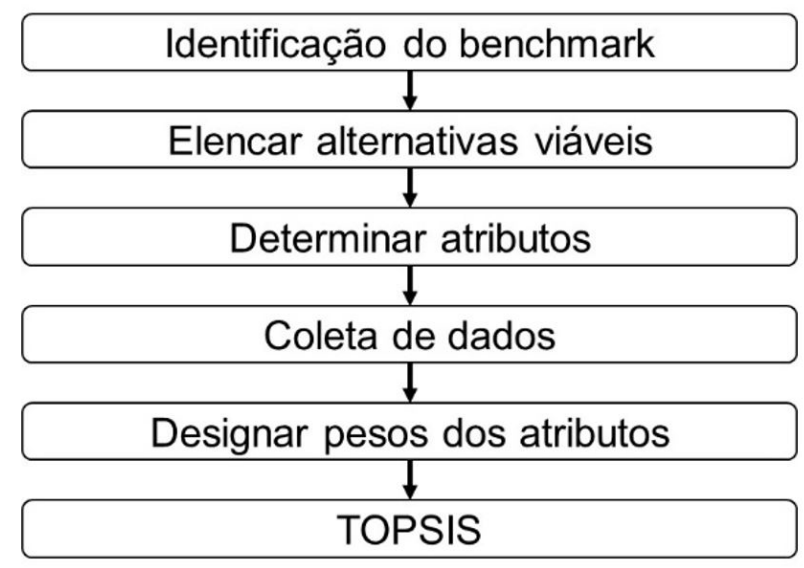

Figura 1. Procedimento para avaliação multiatributo adotado.
Foram selecionados cinco atributos exibidos na Tabela 2 com as suas principais características.

Salienta-se que, entre os atributos, o custo se refere especificamente ao processo em si; todavia existem aqueles que são reflexos ou externalidades gerados pela adoção do processo, que são os casos da redução de emissão da voláteis e a expectativa de contribuição social, os quais são considerados para uma avaliação mais ampla das consequências da decisão de produção, incorporando à análise a preocupação com aspectos de sustentabilidade ambiental e social, respectivamente.

$\mathrm{O}$ atributo porosidade se justifica pela sua relação intrínseca com o processo de fabricação utilizado e a resistência à tração se constitui, do ponto de vista da engenharia, a principal resposta mecânica do compósito produzido, o que, via de regra, define sua aplicabilidade.

$\mathrm{Na}$ etapa de coleta de dados, as medidas 1, 2 e 3 foram coletadas mediante experimentos e as medidas dos atributos 4 e 5, por meio de avaliação subjetiva.

A resina utilizada foi a poliéster insaturada ortoftálica de baixa reatividade, cristal, fabricado pela Embrapol com a denominação comercial ALPHA 190. O peróxido de MEK foi utilizado como agente de cura, na proporção de $1 \%(\mathrm{v} / \mathrm{v})$. A resina de poliéster apresentava, antes da cura, uma viscosidade de 0,48 Pa.s. As fibras de juta foram compradas no comércio de Belém do Pará na forma de fios, com diâmetro médio de $0,62 \mathrm{~mm}$, tramados em tecido no estilo plano (tela), fabricados pela Companhia Têxtil de Castanhal, com gramatura de $225 \mathrm{~g} / \mathrm{m}^{2}$.

Foram fabricadas quatro placas, sendo duas por hand lay-up e duas por infusão a vácuo. As placas fabricadas por hand lay-up foram produzidas, empilhando-se duas camadas do tecido de juta, seguido de laminação manual e cura à temperatura ambiente, sem pressão. Os compósitos produzidos por VIP, foram fabricados, empilhando-se duas camadas do tecido de juta, de acordo com a configuração mostrada na Figura 2 e aplicando-se vácuo de 101,3 kPa, curadas à temperatura ambiente.

A determinação da massa específica e porosidade dos compósitos foram realizadas segundo o procedimento experimental de determinação do empuxo, por imersão em água, conforme recomenda Levy e Pardini [2] e a resistência

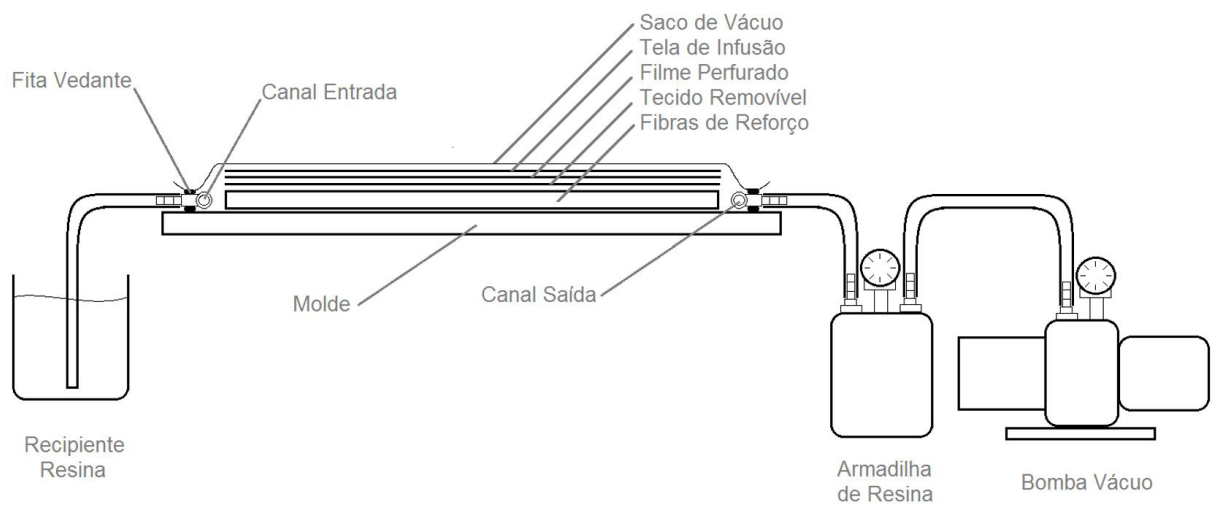

Figura 2. Desenho esquemático da arquitetura utilizada no processo de infusão (VIP) [20]. 
Tabela 3. Escala fundamental de Saaty [18]

\begin{tabular}{|c|c|c|c|c|c|c|c|c|}
\hline \multicolumn{4}{|c|}{ Menos Importante } & \multirow{2}{*}{ Igual } & \multicolumn{4}{|c|}{ Mais Importante } \\
\hline Extremamente & muito forte & Forte & Moderado & & Moderado & Forte & muito forte & Extremamente \\
\hline $1 / 9$ & $1 / 7$ & $1 / 5$ & $1 / 3$ & 1 & 3 & 5 & 7 & 9 \\
\hline
\end{tabular}

a tração conforme a norma ASTM D 3039 [21], realizados em uma máquina de ensaio universal AROTEC WDW 100E, com velocidade de ensaio de $2 \mathrm{~mm} / \mathrm{min}$.

A fração volumétrica do compósito foi previamente fixada em $20 \%$ para os dois processos de fabricação, em função dessa característica interferir diretamente na resposta mecânica de resistência à tração, mantendo entre si forte relação de interdependência. Apesar disto, a fração volumétrica dos fios de juta nos compósitos fabricados por hand lay-up foi de $17,48 \%$ e naqueles fabricados por VIP foi de $20 \%$, devido à diferença de porosidade obtida em cada processo.

Para mensuração das expectativas de contribuição social e para a avaliação de redução de emissão de voláteis, utilizou-se a escala fundamental de Saaty [18] que representa a tradução semântica da preferência de uma alternativa em relação a outra, como apresentado na Tabela 3.

A comparação entre as alternativas é realizada numa matriz, como na Tabela 4 , em que cada elemento, $a_{i j}$, corresponde uma interseção em que cada linha é comparada com a alternativa na coluna por meio da escala da Tabela 3. Por exemplo, a alternativa A1, na primeira linha da matriz da Tabela 4, é julgada por um especialista que atribui um valor na escala de Saaty, $a_{i j}$, que representa a preferência de A1 em relação a alternativa A2, na última coluna.

Na matriz de comparações, $a_{i j}=1 / a_{j i}$ e os valores da diagonal principal serão sempre iguais a 1 , pois representam comparações das alternativas consigo mesmas.

Os escores $\left(\eta_{i}\right)$, que representam a avaliação de cada alternativa, são calculados pela normalização das médias geométricas $\left(g_{i}\right)$ dos elementos das linhas da matriz comparações da Tabela 4, conforme as Equações 9 e 10:

$$
\begin{aligned}
& g_{i}=\sqrt[n]{\prod_{j=l}^{n} a_{i j}} \\
& \eta_{i}=\frac{g_{i}}{\sum_{i=1}^{m} g_{i}}
\end{aligned}
$$

onde $m$ é o total de linhas e $n$ é o total de colunas da matriz de comparações.

Muito embora o TOPSIS permita a designação dos pesos dos atributos subjetivamente, optou-se por uma abordagem objetiva, por meio da entropia da informação [19] uma vez que não se tinha clareza quanto seus valores. A entropia da informação representa a quantidade de informação que cada atributo ou critério disponibiliza na matriz de decisão. Em termos gerais, quanto mais divergentes os valores dos desempenhos em relação a um atributo, maior a sua capacidade discricionária e, consequentemente, maior o peso a lhe ser atribuído.
Tabela 4. Matriz de comparações

\begin{tabular}{ccc}
\hline Alternativas & $\mathbf{A 1}$ & $\mathbf{A 2}$ \\
\hline A1 & 1 & $a_{i j}$ \\
A2 & $1 / a_{j i}$ & 1 \\
\hline
\end{tabular}

Tabela 5. Avaliação das expectativas de contribuição social dos processos de fabricação

\begin{tabular}{ccccc}
\hline & HLU & VIP & $g_{i}$ & $\boldsymbol{\eta}_{i}$ \\
\hline HLU & 1 & 3 & 1,7321 & 0,75 \\
VIP & $1 / 3$ & 1 & 0,5775 & 0,25 \\
Soma & & & 2,3095 & 1,00 \\
\hline
\end{tabular}

Nota: $\mathrm{HLU}=$ processo de laminação manual (Hand Lay-Up); VIP = Processo de Infusão a Vácuo (Vacuum Infusion Process); $\mathrm{g}_{\mathrm{i}}=$ média geométrica dos valores da linha; $\eta_{i}=$ escore.

Assim, a se usar a entropia informacional, os pesos de cada atributo $\left(w_{j}\right)$ foram calculados por meio das Equações de 11 a 14:

$$
\begin{aligned}
& p_{i j}=\frac{x_{i j}}{\max x_{i j}}, i=1, \ldots, m ; j=1, \ldots, n \\
& e_{j}=-\frac{1}{\ln (m)} \sum_{i=1}^{m} p_{i j} \ln \left(p_{i j}\right) \\
& d_{j}=1-e_{j} \\
& w_{j}=\frac{d_{j}}{\sum_{j=I}^{m} d_{j}}
\end{aligned}
$$

onde $x_{i j}$ representa os desempenhos de cada processo relativamente a cada um dos atributos; $p_{i j}$ são os desempenhos normalizados; $d_{j}$, representa o grau de diversidade de informação contida em cada atributo; e $e_{j}$ representa a entropia de cada atributo.

Na última etapa, de posse da matriz de desempenhos e dos pesos de cada atributo, realizou-se avaliação multiatributo entre os processos por meio do método TOPSIS.

Os cálculos foram realizados na Linguagem R [22] por facilitar a automação dos procedimentos, ser aberto e gratuitamente disponível na internet [23] com pacotes específicos para o TOPSIS e para apoiar cálculos de normalização. Os autores disponibilizam a resolução em código em R e em planilha eletrônica por correio eletrônico.

\section{Resultados e discussão}


A Tabela 5 exibe a avaliação das expectativas de contribuição social por meio da comparação pareada, seguindo a escala de Saaty, que compara o elemento da linha em relação ao elemento da coluna. O hand lay-up (HLU), após julgamento realizado por um especialista, foi considerado moderadamente preferível em relação ao VIP (correspondente ao valor 3 na escala Saaty), em virtude daquele exigir um nível de capacitação menor dos operadores o que amplia as possibilidades de contratação de mão de obra. A matriz de comparações com as avaliações atribuídas pelo especialista corresponde às colunas dois e três da Tabela 5. Os valores dos escores foram calculados por meio das Equações $9 \mathrm{e}$ 10 e o melhor desempenho, nesse atributo, foi obtido pelo $\operatorname{HLU}\left(\mathrm{n}_{H L U}=075\right)$.

A avaliação da redução de emissão de voláteis no ambiente pelos processos também foi realizada a partir do parecer de um especialista, utilizando a comparação pareada, seguindo a escala de Saaty [18], formando a matriz correspondente à segunda e terceira colunas da Tabela 6. Esses valores foram convertidos em escores normalizados $\left(\eta_{i}\right)$ para a posterior incorporação à avaliação global, conforme exibido na Tabela 6 .

Nesse caso, tendo em vista que se julgou que o VIP (na segunda linha da matriz) apresenta maior capacidade de retenção de voláteis, quando comparado com o HLU (na coluna), atribuiu-se ao VIP a maior preferência na escala de Saaty [18] (extremamente preferível $=9$ ), resultando no maior escore $\left(\mathrm{n}_{H L U}=0,90\right)$.

Deve-se ressaltar que os valores apresentados na Tabela 6 não se referem diretamente às medidas de redução de emissão de voláteis ao meio ambiente, mas à preferência entre os processos no tocante a esse atributo.

Apesar da atribuição subjetiva da avaliação de atributos conferir um resultado particular à análise, a sua necessidade pode decorrer da natureza qualitativa dessas características; todavia, é importante ressaltar a possibilidade de aplicação do método para apoiar a decisão mesmo que determinadas medidas sejam mensuradas a partir da observação ou opinião de engenheiros. Não obstante, no intuito de reduzir a incerteza

Tabela 6. Avaliação da preferência de nível de redução de voláteis no ambiente pelos processos de fabricação

\begin{tabular}{ccccc}
\hline & HLU & VIP & $\boldsymbol{g}_{\boldsymbol{i}}$ & $\boldsymbol{\eta}_{\boldsymbol{i}}$ \\
\hline HLU & 1 & $1 / 9$ & 0,3333 & 0,10 \\
VIP & 9 & 1 & 3,0000 & 0,90 \\
Soma & & & 3,3333 & 1,00 \\
\hline
\end{tabular}

Nota: $H L U$ = processo de laminação manual (Hand Lay-Up); VIP = Processo de Infusão a Vácuo (Vacuum Infusion Process); $\mathrm{g}_{\mathrm{i}}=$ média geométrica dos valores da linha; $\eta_{i}=$ escore. de tais avaliações, pode-se utilizar a média de avaliações coletadas de um grupo de especialistas.

Pode-se utilizar os escores calculados para os atributos qualitativos definidos neste estudo como valores fixos, evitando-se assim eventuais distorções oriundas da subjetividade do especialista, e coletar as demais medidas normalmente por meio de ensaios, permitindo uma melhor comparação dos resultados e potencializando a reprodução do experimento.

As medidas de desempenho dos processos de produção de compósitos com fios de juta foram organizadas na matriz de desempenho na Tabela 7, nomeadamente, a porosidade, apresentada em unidades absolutas; a resistência à tração, em Megapascal, os custos, em reais, e as avaliações das expectativas de contribuição social e da redução de emissões de voláteis, medidas em escores anteriormente calculados nas Tabelas 5 e 6, respectivamente.

Os resultados demonstraram que o uso do vácuo, no processo VIP, foi eficiente na fabricação de compósitos com mais baixa porosidade e consequentemente maior resistência, em comparação ao processo hand lay-up. Ressalta-se, neste ponto, a pequena variação de fração volumétrica entre os dois processos, apesar dos esforços para fixá-las, que provê pequena vantagem ao processo VIP.

Pode-se constatar, pelo exame inicial da Tabela 7, que a comparação entre os processos é um típico problema multiatributo diante dos vários aspectos, alguns de natureza quantitativas, outros de natureza qualitativa, e que não há relação de dominância entra as alternativas, isto é, uma não é melhor em todos os aspectos do que a outra, uma vez que o processo HLU é mais vantajoso quanto aos custo e contribuição social; por outro lado, o VIP é superior no tocante à porosidade resistência e redução da emissão de voláteis no ambiente.

Outra característica dos problemas multiatributos é a existência de importâncias distintas. Os resultados dos cálculos dos pesos de cada atributo por meio da entropia da informação são apresentados na Tabela 8.

Os atributos com maior ponderação foram a redução da emissão de voláteis no ambiente e porosidade em decorrência de seus maiores graus de diversidade de informação $\left(d_{i}\right)$.

Em virtude de a entropia da informação atribuir as ponderações objetivamente aos atributos, pode haver discordância severa em relação às preferências do tomador de decisão. Nesse caso, sugere-se a atribuição subjetiva de tais valores com apoio de métodos diretos, como a lógica fuzzy, ou de comparações em pares, como o Analytic Hierarchy Process (AHP). Logo, a entropia da informação se revela apropriada neste estudo em face da ausência de

Tabela 7. Matriz de decisão com as medidas de desempenho dos processos de fabricação

\begin{tabular}{cccccc}
\hline Proc. & $\begin{array}{c}\text { Porosidade } \\
\text { (Proporção) }\end{array}$ & Resistência (MPa) & Custo (R\$) & RedVol (Escore) & Social (Escore) \\
\hline HLU & 0,3001 & 17,62 & 79,80 & 0,1000 & 0,75 \\
VIP & 0,0420 & 23,66 & 121,95 & 0,9000 & 0,25 \\
\hline
\end{tabular}

Nota: HLU = processo de laminação manual (Hand Lay-Up); VIP = Processo de Infusão a Vácuo (Vacuum Infusion Process); RedVol = Redução de emissões de voláteis no ambiente. 
Tabela 8. Cálculo dos pesos dos atributos por meio da entropia da informação

\begin{tabular}{cccccc}
\hline & Porosidade & Resistência & Custo & RedVol & Social \\
\hline$e_{j}$ & 0,5373 & 0,9845 & 0,9683 & 0,469 & 0,8113 \\
$d_{j}$ & 0,4627 & 0,0155 & 0,0317 & 0,531 & 0,1887 \\
$w_{j}$ & 0,3763 & 0,0126 & 0,0258 & 0,4318 & 0,1535 \\
\hline
\end{tabular}

Nota: $e_{j}$ representa a entropia de cada atributo; $d_{j}$ é o grau de diversidade de informação; $w_{j}$ representa o peso de cada atributo; RedVol = Redução de emissões de voláteis no ambiente.

Tabela 9. Matriz com dados normalizados $\left(r_{i j}\right)$

\begin{tabular}{cccccc}
\hline & Porosidade & Resistencia & Custo & RedVol & Social \\
\hline HLU & 0,9903 & 0,5973 & 0,5476 & 0,1104 & 0,9487 \\
VIP & 0,1386 & 0,802 & 0,8368 & 0,9939 & 0,3162 \\
\hline
\end{tabular}

Nota: HLU representa o processo de laminação manual (Hand Lay-Up); VIP representa o Processo de Infusão a Vácuo (Vacuum Infusion Process); RedVol = Redução de emissões de voláteis no ambiente.

Tabela 10. Matriz com dados normalizados e ponderados $\left(n_{i j}\right)$

\begin{tabular}{cccccc}
\hline & Porosidade & Resistencia & Custo & RedVol & Social \\
\hline HLU & 0,3727 & 0,0075 & 0,0141 & 0,0477 & 0,1456 \\
VIP & 0,0522 & 0,0101 & 0,0216 & 0,4292 & 0,0485 \\
\hline
\end{tabular}

Nota: HLU representa o processo de laminação manual (Hand Lay-Up); VIP representa o Processo de Infusão a Vácuo (Vacuum Infusion Process); RedVol = Redução de emissões de voláteis no ambiente.

Tabela 11. Medidas de distâncias das soluções ideais, s+e s-

\begin{tabular}{clllllll}
\hline Proc. & Poros. & Resist. & Custo & RedVol & Social & s + & s - \\
\hline HLU & 0,1027 & $7,00 \mathrm{E}-06$ & 0,000 & 0,1455 & 0,0000 & 0,4983 & - \\
VIP & 0,0000 & 0,0000 & $5,60 \mathrm{E}-05$ & 0,0000 & 0,0094 & 0,0974 & - \\
& & & & & & & 0,0974 \\
HLU & 0,0000 & 0,0000 & $5,57 \mathrm{E}-05$ & 0,0000 & 0,0094 & - & 0,4983 \\
VIP & 0,1027 & $6,66 \mathrm{E}-06$ & 0,0000 & 0,1455 & 0,0000 & - & \\
\hline
\end{tabular}

Nota: HLU representa o processo de laminação manual (Hand Lay-Up); VIP representa o Processo de Infusão a Vácuo (Vacuum

Infusion Process); $S_{i}^{+}$é a distância da solução ideal; $S_{i}^{-}$é a distância da solução anti-ideal.

dados disponíveis que expressassem as preferências pelos atributos utilizados.

Os valores dos cálculos do TOPSIS são apresentados nas Tabelas 9 a 12. As Tabelas de 9 a 11 correspondem a etapas intermediárias do método e seus valores foram obtidos pelo uso das Equações de 2 a 7.

Finalmente, os resultados, na Tabela 12, revelaram que o processo VIP apresentou um desempenho superior ao do processo hand lay-up. O escore do VIP é cinco vezes maior que do HLU, decorrente principalmente do melhor desempenho do VIP justamente nos atributos que obtiveram maior ponderação, a porosidade e a redução da emissão de voláteis no ambiente.

Portanto, os resultados sugerem que o processo VIP é preferível quando consideradas juntas as exigências de ordem técnica, econômica, ambiental e social. Deve-se ter em conta, como nos exemplifica Franco [24], na indústria naval, que geralmente a primeira consideração na fabricação de compósitos ainda tem sido o custo, de modo que uma avaliação tradicional teria selecionado o processo HLU como o mais apropriado por gerar o menor custo; no entanto, a avaliação multiatributo foi capaz de incorporar outros aspectos que vêm se tornando cada vez mais relevantes e que vêm gerando restrições, ou mesmo
Tabela 12. Medidas de proximidade dos processos de fabricação e ranking final

\begin{tabular}{ccccc}
\hline Processos & $\mathbf{s}^{+}$ & $\mathbf{s}^{-}$ & $\mathbf{C}_{\mathbf{i}}$ & Ranking \\
\hline HLU & 0,4983 & 0,0974 & 0,1634 & $2^{\text {o }}$ \\
VIP & 0,0974 & 0,4983 & 0,8366 & $1^{\circ}$ \\
\hline
\end{tabular}

Nota: HLU = processo de laminação manual (Hand Lay-Up); VIP = Processo de Infusão a Vácuo (Vacuum Infusion Process) ; s+ = distância da solução ideal; s- = distância da solução anti-ideal e $\mathrm{Ci}$ = medida de proximidade.

proibições, aos processos de fabricação em moldes abertos em decorrência das emissões de voláteis prejudiciais ao ambiente e aos trabalhadores envolvidos, a exemplo da legislação portuguesa.

Deve-se acentuar que, mesmo diante de duas alternativas de processos, a análise não foi trivial, tendo em vista a presença de medidas de difícil comparação, como a contribuição social. Assim, a capacidade da análise multiatributo considerar aspectos que antes eram tratados somente como comentários marginais ou em separado, como a sustentabilidade ambiental e social, é uma vantagem que deve ser ressaltada, pois conduz a uma avaliação mais completa, contemplando as demandas dos diversos atores envolvidos direta ou indiretamente no processo de tomada de decisão. 


\section{Conclusões}

Os resultados indicaram que o processo de infusão a vácuo é a melhor alternativa para a produção de compósitos com fios de juta quando comparado com o hand lay-up, levando-se em conta os atributos e pesos utilizados.

O TOPSIS demonstrou ser simples e capaz de considerar facilmente um bom número de atributos quantitativos e qualitativos, utilizando recursos computacionais básicos; portanto, constituindo-se uma ferramenta de apoio promissora na seleção de processos de materiais.

Por fim, o uso da escala de Saaty foi eficaz na geração de um escore quando são utilizados dados qualitativos coletados da opinião de especialistas, possibilitando a integração de variáveis quantitativas e qualitativas na avaliação objetiva com múltiplos atributos dos processos concorrentes.

\section{Referências}

1 Ashby M. Seleção de materiais no projeto mecânico. 4 ed. Rio de Janeiro: Elsevier; 1996.

2 Levy F No, Pardini LC. Compósitos estruturais: ciência e tecnologia. São Paulo: Edgar Blucher; 2006.

3 Ragondet A. Experimental characterization of the vacuum infusion process [thesis]. Nottingham: University of Nottingham; 2005.

4 Hwang CL, Yoon K. Multiple attribute decision making: methods and applications. New York: Springer-Verlag; 1981.

5 Jahan A, Edwards KL, Bahraminasab M. Multi-criteria decision analysis for supporting the selection of engineering materials in product design. Oxford: Butterworth-Heinemann; 2016.

6 Milani AS, Shanian A, Madoliat R, Nemes JA. The effect of normalization norms in multiple attribute decision making models: a case study in gear material selection. Structural and Multidisciplinary Optimization. 2005;29(4):312-318.

7 Shanian A, Savadogo O. TOPSIS multiple-criteria decision support analysis for material selection of metallic bipolar plates for polymer electrolyte fuel cell. Journal of Power Sources. 2006;159(2):1095-1104.

8 Govindan K, Madan Shankar K, Kannan D. Sustainable material selection for construction industry: a hybrid multi criteria decision making approach. Renewable \& Sustainable Energy Reviews. 2016;55:1274-1288.

9 Shanian A, Savadogo O. A methodological concept for material selection of highly sensitive components based on multiple criteria decision analysis. Expert Systems with Applications. 2009;36(2):1362-1370.

10 Chauhan A, Vaish R. Magnetic material selection using multiple attribute decision making approach. Materials \& Design. 2012;36:1-5.

11 Chauhan A, Vaish R. A comparative study on decision making methods with interval data. Journal of Computational Engineering. 2014;2014:1-10.

12 Karande P, Chakraborty S. Application of multi-objective optimization on the basis of ratio analysis (MOORA) method for materials selection. Materials \& Design. 2012;37:317-324.

13 Çalışkan H, Kurşuncu B, Kurbanoğlu C, Güven ŞY. Material selection for the tool holder working under hard milling conditions using different multi criteria decision making methods. Materials \& Design. 2013;45:473-479.

14 Anojkumar L, Ilangkumaran M, Sasirekha V. Comparative analysis of MCDM methods for pipe material selection in sugar industry. Expert Systems with Applications. 2014;41(6):2964-2980.

15 Mansor MR, Sapuan SM, Hambali A, Zainudin ES, Nuraini AA. Materials selection of hybrid bio-composites thermoset matrix for automotive bumper beam application using TOPSIS method. Advances in Environmental Biology. 2014;(8):3138-3142.

16 Mansor MR, Sapuan SM, Zainudin ES, Nuraini AA, Hambali A. Application of integrated AHP-TOPSIS method in hybrid natural fiber composites materials selection for automotive parking brake lever component. Australian Journal of Basic and Applied Sciences. 2014;8(5):431-439.

17 Al-Oqla FM, Sapuan SM, Ishak MR, Nuraini AA. Decision making model for optimal reinforcement condition of natural fiber composites. Fibers and Polymers. 2015;16(1):153-163.

18 Saaty TL. The analytic hierarchy process: planning, priority, setting and resource allocation. New York: McGrawHill; 1980.

19 Shannon CE, Weaver W. A mathematical theory of communication. The Bell System Technical Journal. 1948;27(3):379-423. 
Aplicação do TOPSIS na análise do processo de infusão a vácuo para fabricação de compósitos com fios de juta

20 Rodrigues J, Souza JA, Fujiyama R. Compósitos poliméricos reforçados com fibras naturais da Amazônia fabricados por infusão. Revista Matéria. 2015;20(4):946-960.

21 American Standard Test Methods. ASTM D3039: standard test method for tensile properties of polymer matrix composite materials. West Conshochen: ASTM; 2008.

22 R Core Team. R: a language and environment for statistical computing. Vienna: R Foundation for Statistical Computing; 2018 [acesso em 28 maio 2019]. Disponível em: https://www.R-project.org/

23 R Core Team. CRAN: the comprehensive archive network. Vienna: R Foundation for Statistical Computing; 2018 [acesso em 28 maio 2019]. Disponível em: https://www.r-project.org/

24 Franco RAVS. Produção de componentes em materiais compósitos por infusão de resina [dissertação]. Lisboa: Universidade Técnica de Lisboa; 2008.

Recebido em: 28 Maio 2019

Aceito em: 15 Maio 2020 